Dear Author,

Please, note that changes made to the HTML content will be added to the article before publication, but are not reflected in this PDF.

Note also that this file should not be used for submitting corrections. 


\title{
Experimental determination of the streaming potential across cation-exchange membranes with different morphologies
}

\author{
P. Somovilla, J.P.G. Villaluenga*, V.M. Barragán, M.A. Izquierdo-Gil \\ Department of Applied Physics I, University Complutense of Madrid, 28040 Madrid, Spain
}

\section{A R T I C L E I N F O}

Article history:

Received 28 July 2015

Received in revised form

28 October 2015

Accepted 22 November 2015

Keywords:

Electro-kinetic phenomena

Non-equilibrium thermodynamics

Cation-exchange membranes

\begin{abstract}
A B S T R A C T
Liquid uptake and streaming potential have been determined in aqueous sodium chloride solutions for five different commercial sulfonated polymer cation-exchange membranes. The selected membranes have distinct morphologies and electrochemical properties. Differences in the liquid uptake properties of the membranes have been found, which have been analysed on the basis of the structure and the chemical properties of the membranes. In most of the membranes analyzed, the higher the liquid content of the membranes, the lower the effective concentration of fixed charges in the membranes. The streaming potential across the membranes increases linearly with the established pressure difference, and it is larger in heterogeneous membranes than in homogeneous ones. In general, the higher the membrane liquid content, the higher the streaming potential across the membranes.
\end{abstract}

(c) 2015 Published by Elsevier B.V.

\section{Introduction}

When an ion-exchange membrane separating two electrolyte aqueous solutions is subject to one or more driving forces, transport phenomena of electro-kinetic character can be originated through the membrane. For instance, a pressure difference across a membrane is able to drive both a volume flux and an electric charge flux through the membrane. This electro-kinetic phenomenon indicates that mass and electric charge transport processes can be coupled. Coupling means that a mass flux can be caused by an electric potential difference, and that an electric charge flux can be originated by a pressure difference alone.

If an excess hydrostatic pressure is applied to the solution on one side of an ion-exchange membrane, the liquid is forced to move across the membrane along the pressure difference. This liquid carries along a net electric charge, as a consequence of the excess of counter-ions with respect to the co-ions, towards the low-pressure membrane side, which acquires the same charge as the counter-ions, while the high-pressure membrane side acquires the charge of the fixed groups. Accordingly, an electric potential difference appears between the two sides of the membrane, which is known as streaming potential.

The streaming potential acts over the electrically charged liquid inside the membrane, partly balancing the effect of the pressure and thus reducing the flow across the membrane. The streaming potential also accelerates the co-ions and slows down the counter-

\footnotetext{
* Corresponding author.

E-mail address: jpgarcia@ucm.es (J.P.G. Villaluenga).
}

ions in such a way that, despite their different concentrations, both of them transfer equivalent amounts of electric charge in the same time. However, the effect of applied pressure is stronger than the one produced by the developed electric field and thus, relative to the membrane matrix; both ionic species are carried out along with the solvent flux.

According to the linear non-equilibrium thermodynamics approach, the phenomenological equations for modelling the transport of mass and electric charge across an ion-exchange membrane are [1]

$J_{V}=L_{11} \Delta P+L_{12} \Delta \psi$

$I=L_{21} \Delta P+L_{22} \Delta \psi$

where $J_{V}$ is the volume flux, $I$ is the electric current, $\Delta P$ is the pressure difference across the membrane, and $\Delta \psi$ is the difference of electric potential across the membrane. The coefficients $L_{11}$ and $L_{22}$ are the hydraulic permeability and the electric conductance, respectively, whereas $L_{12}$ and $L_{21}$ are the electro-kinetic coefficients that take into account the coupling phenomenon.

Coupling can be studied in terms of the so-called pressure coefficient $\beta$ and electro-osmotic permeability $W$, which can be defined, from Eqs. (1) and (2), as follows:

$$
\beta \equiv\left(\frac{\Delta \psi}{\Delta P}\right)_{I=0}=-\frac{L_{21}}{L_{22}}
$$

0376-7388/0.010/10.1016/j.memsci.2015.11.030 
$W \equiv\left(\frac{J_{V}}{I}\right)_{\Delta P=0}=\frac{L_{12}}{L_{22}}$

The Onsager's reciprocity relation implies that $L_{12}=L_{21}$, it then follows that $W=-\beta$. This relation, which can be expressed as

$\left(\frac{J_{V}}{I}\right)_{\Delta P=0}=-\left(\frac{\Delta \psi}{\Delta P}\right)_{I=0}$

is known as Saxén's law.

In order to determine experimentally the streaming potential, a pressure difference between the two sides of an ion-exchange membrane is established, and the electric potential difference $\Delta \psi$ across the membrane is measured as a function of time $t$. A typical plot of $\Delta \psi$ vs. $t$ shows an initial sudden rise in the electric potential difference, when $\Delta P \neq 0$, which represents the potential arising from the pressure difference, when the two bulk solution concentrations are equal. This is the electrical potential difference, $\Delta \psi_{0}$, in the absence of concentration polarization because there is no liquid flow through the membrane in this stage. As the process takes place, a concentration difference between the two sides of the membrane is also originated. It has been found [2] that this term is proportional to $t^{1 / 2}$. Thus, the contribution of the concentration polarization to the electric potential difference can be removed by determining the streaming potential as an intercept at $t=0, \Delta \psi_{0}$ from the plot of the experimental curves of $\Delta \psi$ vs. $t^{1 / 2}$, i.e., the streaming potential is obtained in the initial time of the diffusion step

$-\Delta \psi=-\Delta \psi_{0}+A t^{1 / 2}$

where the constant $A$ can be related to the hydrodynamic permeability in the membrane [3].

The value of the streaming potential $\Delta \psi_{0}$ found as explained above does not coincide with the real value. The use of reversible electrodes involves the correction of the measured potential due to the pressure difference. When an electrode $\mathrm{Ag} / \mathrm{AgCl}$ is used, the true streaming potential $\Delta \psi_{r}$ can be obtained by subtracting a contribution from the experimentally determined value as follows:

$\Delta \psi_{r}=\Delta \psi_{0}-\frac{1}{F}\left(v_{\mathrm{AgCl}}-v_{\mathrm{Ag}}\right) \Delta P$

where $F$ is Faraday's constant, and $v_{\mathrm{AgCl}}$ and $v_{\mathrm{Ag}}$ are the molar volumes of $\mathrm{AgCl}$ and $\mathrm{Ag}$, respectively [4].

This work is a contribution of the application of linear nonequilibrium thermodynamics theory to the study of the transport processes in charged membranes. This work is concerned to the search of relations between polymer membrane properties and morphology. With this aim, the liquid uptake and the streaming potential have been experimentally determined for several cationexchange membranes with different structures and electrochemical properties.

\section{Experimental}

\subsection{Membrane and materials}

The main characteristics of the commercial cation-exchange membranes used in this study are given in Table 1. Nafion-115 membranes (hereafter named NF115) are films consisted of a polytetrafluorethylene backbone and long fluorovinyl ether pendant side chains regularly spaced, terminated by a sulphonate ionic groups. There are no cross-links between the polymers. MK-40 membranes (hereafter named MK40) are composites formed from the cation-exchange resins KU-2 (polystyrene matrix cross-linked
Table 1

Ion exchange capacity (IEC), density $\left(\rho_{m}\right)$, thickness $\left(d_{m}\right)$, water content $\left(s_{w}\right)$, wet membrane porosity $\left(\varepsilon_{w}\right)$, FH interaction parameter $\left(\chi_{w}\right)$, and parameter $\lambda$ in water of cation-exchange membranes used in this study.

\begin{tabular}{llllllll}
\hline Membrane & IEC $(\mathrm{meq} / \mathrm{g})^{\mathrm{a}}$ & $\rho_{m}\left(\mathrm{~g} / \mathrm{cm}^{3}\right)^{\mathrm{a}}$ & $d_{m}(\mu \mathrm{m})^{\mathrm{a}}$ & $s_{w}{ }^{\mathrm{b}}$ & $\varepsilon_{w}{ }^{\mathrm{c}}$ & $\chi_{w}{ }^{\mathrm{d}}$ & $\lambda^{\mathrm{e}}$ \\
\hline MK40 & 2.6 & 1.12 & 480 & 0.53 & 0.37 & 0.91 & 11 \\
CR65 & 2.3 & 0.877 & 550 & 0.56 & 0.33 & 0.98 & 14 \\
RXC & 2.2 & 0.817 & 650 & 0.64 & 0.34 & 0.96 & 16 \\
CMX & 1.7 & 1.0 & 170 & 0.30 & 0.23 & 1.18 & 10 \\
NF115 & 1.0 & 2.0 & 130 & 0.20 & 0.29 & 1.05 & 11 \\
\hline
\end{tabular}

a Provided by the manufacturer. IEC (meq/g dry membrane); $\rho_{m}\left(\mathrm{~g} / \mathrm{cm}^{3}\right)$ dry membrane.

${ }^{b}$ Estimated from Eq. (8).

c Estimated from Eq. (12)

d Estimated from Eq. (13).

e Estimated from Eq. (9).

with divinylbenzene and fixed groups), polyethylene and nylon. Membranes CR65-CZL-412 (hereafter named CR65) are crosslinked sulfonated copolymers of vinyl compounds cast in homogeneous films on synthetic reinforced fabrics. Neosepta CMX membranes (hereafter named CMX) are composites prepared on the base of polystyrene and divinylbenzene, and reinforced with polyvinylchloride. Ralex CMH-PES membranes (hereafter named RXC) are composites formed from ion-exchange resins, polyethylene and polyamide as the reinforcing material. The membranes can be divided with respect to their structure and preparation into two categories: MK40, CR65 and RXC are considered as heterogeneous membranes, whereas NF115 and CMX are considered as homogeneous membranes. Based on the type of fixed charge group, the five membrane-types are classified as strong acid base membranes because contain sulphonate groups as charged group.

\subsection{Liquid uptake measurements}

In order to determine the liquid uptake by the membrane, a liquid swelling study was performed by using a water solution with a concentration of $0.01 \mathrm{M} \mathrm{NaCl}$. Before the experiments, the membrane samples were dried under vacuum at $30.0 \pm 0.1^{\circ} \mathrm{C}$ for $24 \mathrm{~h}$ and weighted. After that, the samples were immersed in a closed bottle containing the solution and allowed to equilibrate. The bottle was placed in an oven thermostatted at $25.0 \pm 0.1^{\circ} \mathrm{C}$. After several days of immersion, when no more liquid is sorbed, the swelling is considered to be completed and the swollen membrane was taken out of the liquid, wiped carefully with filter paper and weighted again. The increase in weight was equal to the weight of the liquid sorbed by the membrane. The liquid uptake was estimated from the weight of the swollen and the dry membrane sample according to the following expression:

$s=\frac{m_{w}-m_{d}}{m_{d}}$

where $m_{w}$ and $m_{d}$ are the weights of the swollen and dry membrane, respectively. The water content of the membrane can be expressed as the average number of water molecules per conducting functional group, $\lambda$, determined by [5]

$\lambda=\frac{S}{I E C \cdot M_{w}}$

where IEC represents the ion-exchange capacity (i.e. the ionizable hydrophilic functional groups content per gram of polymer, eq/g) and $M_{w}$ is the molecular weight of water. 


\subsection{Streaming potential measurements}

The experimental device and the procedures used in this study were essentially the same as those described in [6]. Basically, the cell consisted of two glass chambers of approximately $125 \mathrm{~cm}^{3}$ each, connected by a rigid threaded joint, where the membrane was inserted between two plane O-rings. Each half-cell contained water solution with a concentration of $0.01 \mathrm{M} \mathrm{NaCl}$. Each half-cell included an inlet, in which an $\mathrm{Ag} / \mathrm{AgCl}$ electrode was introduced. In one of the chambers there was another inlet, in which a pressure connector was introduced. The pressure pulses were generated with pure pressurized air through a double pressure reducer. Pulses up to to 1 bar were applied in this half-cell. The low pressure half-cell was always kept at atmospheric pressure. The pressure difference was also measured with a digital barometer with $68.9 \mathrm{~Pa}(0.01 \mathrm{psig})$ resolution.

The acquisition of streaming potential data was made by means of an HP 34401 logging multimeter with a high resolution, recording the data vs. time. They were corrected for possible electrode asymmetry. The electrical device was inside a Faraday box to prevent undesired electrical perturbations. The electrode immersed in the lower pressure solution was permanently grounded. The whole cell was immersed in a thermostatted bath at $25.0 \pm 0.1^{\circ} \mathrm{C}$.

Before experiments, the membrane was immersed in aqueous solution with a concentration of $0.01 \mathrm{M} \mathrm{NaCl}$ for a minimum of $48 \mathrm{~h}$ in order to achieve equilibrium.

\section{Results and discussion}

\subsection{Liquid uptake}

The estimated liquid uptakes of the membranes in $\mathrm{NaCl} /$ water solutions are given in Table 2. All values were measured three times and the errors are less than 5\%. In general, the liquid uptake values obtained in the present study are in a good agreement with the data for $0.01 \mathrm{M} \mathrm{NaCl}$ aqueous solutions published in the literature. The small differences are probably due to differences in the chemical or thermal treatments of the membranes. We obtained a liquid uptake of 0.67 for MK40, whereas Larchet et al. [7] reported a value of 0.52 . We obtained a value of 0.11 for liquid uptake by NF115, and the solvent content of Nafion-type membranes ranges from 0.06 to 0.20 , depending on the ionic form of the membrane and the concentration of external solution [8-10]. We obtained a liquid uptake of 0.29 for CMX, whereas the data reported in the literature are 0.31 [11], 0.25 [12] and 0.18 [13]. We

Table 2

Liquid uptake $(s)$, concentration of fixed ion group per volume of swollen gel $(\bar{X})$, effective fixed charge concentration $\left(\bar{X}_{\text {eff }}\right)$, parameter $r$, wet membrane porosity $(\varepsilon)$,

fixed charge concentration $\left(\bar{X}^{*}\right)$, and $\mathrm{FH}$ interaction parameter $(\chi)$ in $\mathrm{NaCl} 0.01 \mathrm{M}$ solution, of cation-exchange membranes used in this study.

\begin{tabular}{llllllll}
\hline Membrane & $s^{\mathrm{a}}$ & $\bar{X}^{\mathrm{b}}(\mathrm{mol} / \mathrm{l})$ & $\bar{X}_{\text {eff }}{ }^{\mathrm{c}}(\mathrm{mol} / \mathrm{l})$ & $r$ & $\varepsilon^{\mathrm{d}}$ & $\bar{X}^{* \mathrm{e}}(\mathrm{mol} / \mathrm{l})$ & $\chi^{\mathrm{f}}$ \\
\hline MK40 & 0.67 & 0.91 & 0.53 & 0.58 & 0.43 & 1.60 & 0.84 \\
CR65 & 0.64 & 0.69 & 0.30 & 0.43 & 0.36 & 1.10 & 0.93 \\
RXC & 0.62 & 0.65 & 0.26 & 0.40 & 0.34 & 0.99 & 0.97 \\
CMX & 0.29 & 1.16 & 0.31 & 0.27 & 0.23 & 1.51 & 1.19 \\
NF115 & 0.11 & 1.74 & 0.35 & 0.20 & 0.18 & 2.12 & 1.33 \\
\hline
\end{tabular}

\footnotetext{
a Estimated from Eq. (8).

b Estimated from Eq. (14).

${ }^{\mathrm{c}}$ Estimated from Eqs. (15) and (16).

d Estimated from Eq. (12).

e Estimated from $\bar{X}$ and $\varepsilon$.

${ }^{f}$ Estimated from Eq. (13).
}

obtained a liquid uptake of 0.62 for RXC, whereas the reported value is 0.31 [13].

Heterogeneous membranes (MK40, CR65, RXC) show higher solution uptakes than homogeneous membranes (CMX and NF115), indicating that heterogeneous membranes are more hydrophilic, in agreement with previous results [14]. In general, the structure of the heterogeneous membranes consists of small ionexchanger particles dispersed distributed in an inert binder. The ion-exchange particles are in contact with one another, and it is reported that most of heterogeneous membranes contain about $50-75$ vol\% of ion-exchange material. As the binder is highly hydrophobic, the swelling of membrane matrix in water is expected to be very low. Then, the amount of liquid within the membrane is determined by the degree of swelling of the ion-exchanger particles, and the volume fraction of inter-particle voids formed by the swelling or contracting particles. On the one hand, water interacts strongly with the particles due to its higher polarity; on the other hand, it is easy to accommodate water molecules in the sorption sites located in the particles due to the small molecular size of water. These facts would explain the high liquid sorption degree in MK40, CR65 and RXC. Usually, the polymer backbone is highly hydrophobic in homogeneous membranes, whereas the sulphonic groups are very hydrophilic. In the presence of a polar solvent, such as water, this gives rise to some hydrophobic/hydrophilic separation. Water molecules reside completely in the hydrophilic domains of the polymer, because water is excluded from the hydrophobic backbone. These facts would explain the lower sorption value of liquid in NF115 and CMX. On the other hand, as can be seen from data in Table 1 , the $\lambda$ parameter is not directly related to the IEC. As the membranes have the same sulphonic ion group, this fact may be related to differences in the structure of the membranes, which may affect the chemical affinity between the membrane polymer and the water.

Since the concentration of electrolyte is quite low, the liquid uptake properties of the membranes should not be essentially different in water and in the $0.01 \mathrm{M} \mathrm{NaCl}$ solution, as the comparison of the liquid content data in Tables 1 and 2 reveals. It is well known that with increasing electrolyte concentration, the membrane liquid content decreases [15]. The reason is that with increasing concentration of external solution the osmotic pressure increases, while that in the internal solution of the membrane grows only to a small extent, since the ionic force changes a little. This is the behaviour exhibited by the NF115 membrane, whose liquid uptake decreases to a $60 \%$ in the presence of the salt. In contrast, when the other membranes are equilibrated with a $0.01 \mathrm{M} \mathrm{NaCl}$ solution, the liquid uptake either remains unchanged, such as for RXC and CMX, or increases a little, such as for MK40 (23\%) and CR65 (13\%), compared to the membranes equilibrated with pure water. This behaviour can be attributed to the either the high cross-linking degree or the reinforcement of these membranes, and thus to their high mechanical stability [16]. This is in accordance with previous results with the same membranes [14], in which it was observed that the influence of the presence of salt on solution was different for membranes with different structures. Thus, membranes with a high density and a deformable morphology, such as NF115, the presence of the electrolyte can play an influential role in the membrane uptake properties. In contrast, the effect of the electrolyte is less noticeable in rigid membranes with low polymer elasticity, such as MK40, CR65, RXC and CMX.

It is known that part of the liquid sorbed by the membrane is due to the solvation of the membrane ionized fixed groups, therefore liquid content would be favoured by a high ion-exchange capacity of the membrane. Based on this fact, it would be expected that the higher the IEC of the membrane, the higher the solution uptake. However, the structure of the polymeric matrix is also an important factor, and it is expected that membranes with lower 
density present higher porosity and higher liquid sorption. Data in Tables 1 and 2 are in reasonable good agreement with this statement. Thus, RXC membrane, with lower density presents higher liquid content than the other heterogeneous membranes.

The membrane wet porosity $\varepsilon$ (volume of free solution within the membrane per unit volume of wet membrane) can be defined as follows:

$\varepsilon=\frac{\Delta V}{(1+\Delta V)}$

where $\Delta V$ designates the volume increase of the membrane upon absorption of the electrolyte solution per unit of dry membrane volume. This quantity was calculated for membranes with both water and electrolyte solution content data using the following equation:

$\Delta V=\frac{\left(m_{w}-m_{d}\right) \rho_{m}}{m_{d} \rho_{l}}$

where $\rho_{m}$ and $\rho_{l}$ are the densities of the dry membrane and the sorbed liquid, respectively. Combining Eqs. (8), (10) and (11), the porosity can be expressed as a function of the liquid uptake $s$ as follows:

$\varepsilon=\left[1+\frac{\rho_{l}}{s \rho_{m}}\right]^{-1}$

The analysis of the sorption properties of the membranes can also be made using a model derived from the Flory-Huggins (FH) theory [17], which makes a statistical calculation of the different configurations of a polymer/liquid system upon some assumptions on molecular shapes and interactions. The model enables sorption modelling using a single parameter, the so-called FH interaction parameter, which is a reflection of the polymer/liquid affinity. The lowest FH parameter values describe the highest liquid sorption level and, thus the best affinities. The FH interaction parameter can be estimated as

$\chi=-\frac{\ln \varepsilon+(1-\varepsilon)}{(1-\varepsilon)^{2}}$

The estimated values of the porosity and the $\mathrm{FH}$ interaction parameter for membrane/water system are given in Table 2. A value $0.99749 \mathrm{~g} / \mathrm{cm}^{3}$ has been used as solution density at $25^{\circ} \mathrm{C}$ [18]. It is found that the wet porosity of heterogeneous membranes is larger to a small extent than that of homogeneous membranes. In addition, the $\mathrm{FH}$ parameters for heterogeneous membranes are lower than that of homogeneous membranes. Both facts suggest better polymer/water affinities for the heterogeneous membranes than those of homogeneous membranes.

The calculated values of the membrane wet porosity in $0.01 \mathrm{M}$ $\mathrm{NaCl} /$ water solutions are also given in Table 2 . A value $0.99749 \mathrm{~g} /$ $\mathrm{cm}^{3}$ has been used as solution density at $25^{\circ} \mathrm{C}[18]$. In general, the effect of the presence of the electrolyte in the porosity is small and it seems to be independent of the heterogeneous or homogeneous character of the membrane. It is found that the porosity in water and in the solution is nearly the same for membranes CR65, RXC and CMX. In contrast, in presence of the electrolyte, NF115 porosity decreases $37 \%$, and MK40 increases its porosity in a 16\%. The low solution concentration and the great selectivity of the membranes assure the almost complete exclusion of co-ions inside of the membrane. Then, it can be considered that membranes only absorb water and Eq. (13) can be used to estimate the FH parameter. The corresponding values are also reported in Table 2. Clearly, the changes in the $\mathrm{FH}$ interaction parameter are in accordance with the changes in the liquid content of the membrane.

As noted above, the swelling of an ion-exchange membrane results from the balance between the internal osmotic pressure and the forces associated with the elasticity of the polymeric matrix. The ion-exchange capacity, indicating the density of ionizable hydrophilic functional groups in the membrane, strongly affects the ion transfer. However, IEC values are not particularly useful for characterizing membrane performance because it does not take into account the swelling properties of the polymer. A more appropriate measure of the true concentration of fixed charge groups is the concentration of the fixed ions groups of the membrane per volume of swollen membrane, $\bar{X}$ [16]. The value of this variable can be determined from the ion-exchange capacity given by the manufacturer, the density and the liquid uptake of the membrane [15] as follows:

$$
\bar{X}=\rho_{m} \frac{100-s}{100\left(1+\sum_{i} Q_{i}-M_{r e f} 10^{-3} I E C\right)} \text { IEC }
$$

where $\bar{X}$ is the molarity of fixed groups (in equivalents per cubit decimetre), $\rho_{m}$ is the density of membrane (in grams per cubic centimetre), $s$ is the liquid content of the membrane (in weight per cent), $Q_{i}$ is a dimensionless variable which expresses the amount of species $i$ in the "specific amount" of membrane, and $M_{r e f}$ is the atomic weight of the reference ion ( 1 for cation exchangers). The summation is carried out over counter-ions and sorbed solutes, if any. This fixed charge concentration depends on the liquid uptake properties, and thus it will be different for different membranes and solutions. The estimated values of fixed charge concentration $\bar{X}$ are given in Table 2. As can be observed, homogeneous membranes have higher concentration of fixed charges than heterogeneous membranes, but lower IEC values, due to their lower liquid content. The concentration of fixed charges $\bar{X}$ decreases in the following order: NF115 $>$ CMX $>$ MK40 $>$ CR65 $>$ RXC. It should be pointed out that the interstitial void fraction in the membranes is not included in Eq. (14). The concentration of fixed ion group, when the porosity of the membranes is considered, can be estimated as $\bar{X}^{*}=\bar{X}(1-\varepsilon)^{-1}[19]$. The results for $\bar{X}^{*}$ are also given in Table 2 . The parameter $\bar{X}^{*}$ decreases in the following order: NF115 $>$ MK40 $>$ CMX $>$ CR65 $>$ RXC. The parameters $\bar{X}$ and $\bar{X}^{*}$ follow the same sequence with the exception of MK40. This fact may be related to the high porosity of this membrane. On the other hand, it is noted that the values of $\bar{X}^{*}$ are in a reasonable accordance with the results obtained by other authors. For example, Belashova et al. [20] reported a value of $1.7 \mathrm{~mol} / \mathrm{dm}^{3}$ for the MK40 membrane in aqueous $0.02 \mathrm{M} \mathrm{NaCl}$ solution, and Lehmani et al. [19] reported a value of $1.26 \mathrm{~mol} / \mathrm{dm}^{3}$ for the Nafion 117 membrane with $0.01 \mathrm{M} \mathrm{NaCl} /$ water solution.

The effectiveness of fixed charge in sulfonated polysulfone membranes is strongly correlated with the physicochemical properties [21]. The effective value of fixed charge is always lower than the corresponding concentration of ionic groups, indicating that not all of the fixed charge groups play an active role in the ion transport. As a general trend, an increase of the effective charge with the liquid uptake is observed. This is due to the low liquid uptakes favour the interaction between the counter-ion and the charged groups in the polymer, and so, a decrease of the effective charge of the membrane. The effective charge concentration, $\bar{X}_{\text {eff }}$, for a given charged polymer membrane can be estimated when its density, liquid uptake and concentration of the fixed ionic groups per volume of swollen membrane are known, using the following equations [14]:

$\bar{X}_{e f f, 1}=\frac{3.68 \rho_{r} s \bar{X}}{N_{h}}$

$\bar{X}_{e f f, 2}=0.78 \rho_{r} s \bar{X}$ 
where $\rho_{r}$ is the membrane density relative to the water density, and $N_{h}$ the hydration number of the cation. Barragán et al. [14] stated that Eq. (15) is valid for dense polymer membranes, with low liquid content, with influence of the electrolyte on the liquid uptake properties. In contrast, Eq. (16) is valid for more porous polymer membranes, with high liquid content, for which the influence of the electrolyte is low. The estimated values of $\bar{X}_{\text {eff }}$ are collected in Table 2 . The effective charge concentration $\bar{X}_{\text {eff }}$ decreases in the following order: MK40 $>$ NF115 $>$ CMX $>$ CR65 $>$ RXC. It is worth mentioning that the value is significantly larger for MK40 than that of other membranes. Moreover, it is also found that the parameters



C

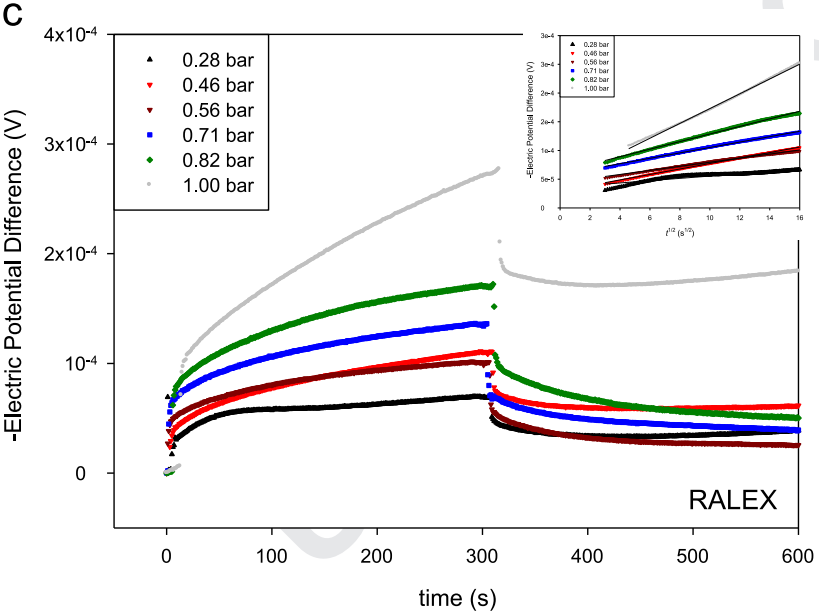

$\bar{X}^{*}$ and $\bar{X}_{\text {eff }}$ follow the same sequence with the exception of MK40. The interaction between the counter-ions and the fixed charged groups in the membranes is higher for less swollen membranes [14]. At low liquid content, the ion-pairs between the fixed charge groups and counter-ions in the membrane occur, originating a charge effectiveness decrease. Moreover, when the membrane porosity is high, it seems reasonable to accept that the ion-pairing counter-ions and the fixed ionic groups are scarcer. Thus, the MK40 exhibits the largest effective charge concentration with a little ionpairing effect because this membrane combines a high degree of

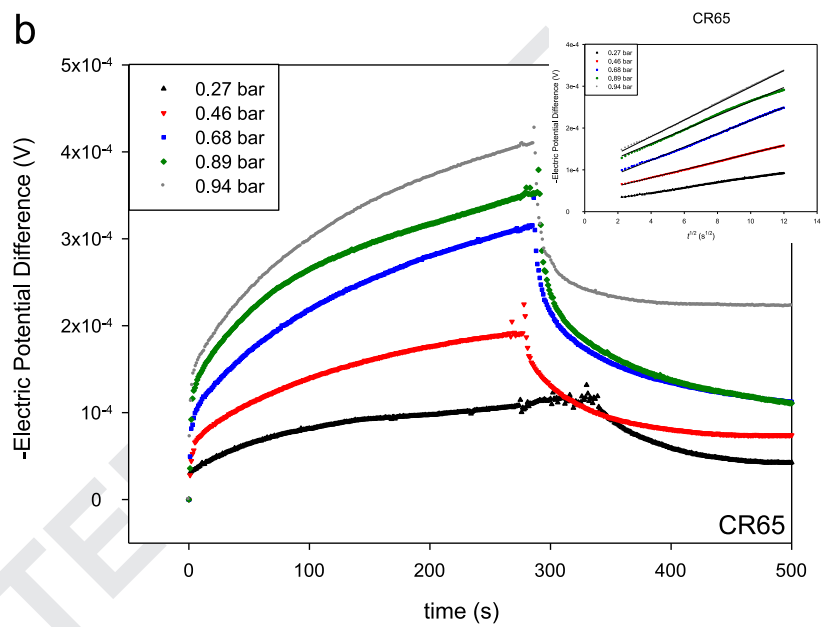

d


process is hindered because the encounters between the mobile

e

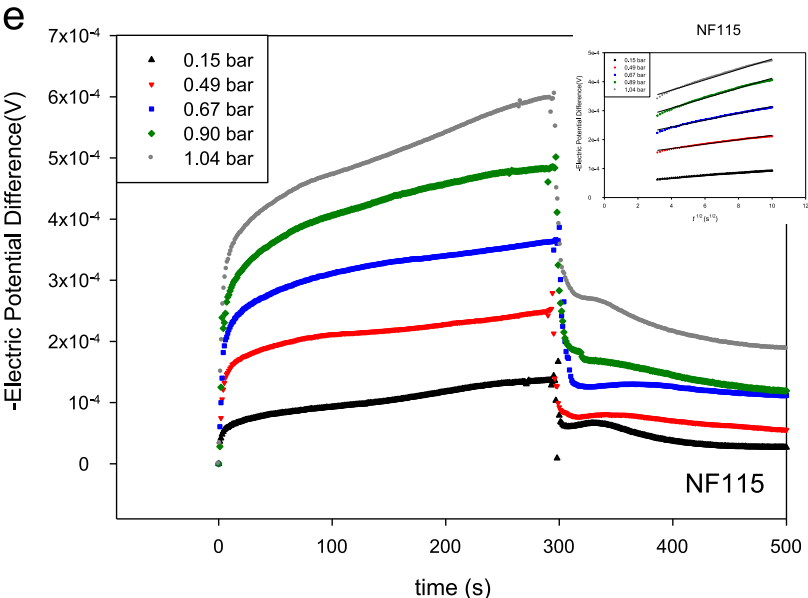

Fig. 1. Electric potential difference originated by a pressure difference across the membrane as a function of time: (a) MK40, (b) CR65, (c) RXC, (d) CMX, and (e) NF115. 
swelling with a high porosity.

The effective value of the fixed charge is lower that the corresponding concentration of fixed ionic groups, indicating that not all of the fixed charged groups plays an active role in the ion transport. The ratio between both charge concentrations, $r=\bar{X}_{\text {eff }} / \bar{X}$, is also reported in Table 2. This selectivity parameter is an important parameter for practical applications because it is both a measure of the charge effectiveness and an indicative of the selectivity loss of the membrane in a given medium. The parameter $r$ decreases in the following order: MK40 $>$ CR65 $>$ RXC $>$ CMX $>$ NF115. The results indicate that heterogeneous membranes have higher $r$ values than those of homogeneous membranes. Otherwise, the results suggest that there is a correlation between the parameter $r$ and the FH interaction parameter $\chi$, because the higher $r$ the lower $\chi$. This fact implies that a better affinity between the polymer and the absorbed solution leads to a lower selectivity loss in the membrane.

\subsection{Streaming potential}

The electric potential difference that appears when a pressure difference is established between the two sides of an ion-exchange membrane was measured as a function of time. Measurements were carried out for several pressure difference values between 0 and $10^{5} \mathrm{~Pa}$ with $0.01 \mathrm{M} \mathrm{NaCl}$ aqueous solutions. It should be noted that each series of measurements started with the highest pressure difference. The results, which are shown in Fig. 1a-e, are in agreement with the expected behaviour [22,23]. As it is expected, the electric potential difference has an opposite sign than the pressure difference, and it increases with the established pressure difference.

In general, the curves are qualitatively similar in all cases. When the pressure difference is applied, the electric potential increases rapidly within a few seconds followed by a slower increase. Approximately at a time of $300 \mathrm{~s}$, when the pressure difference across the membrane was removed, the potential decreases rapidly within a few seconds followed by a slow decrease to a constant value. In contrast, the behaviour exhibited for membrane MK40 at short times is a little bit different. It was observed that after the sharp increase when pressure difference is applied, the potential decreases slowly with time within a few seconds, and then it increases in a similar manner than that of the other membranes. This decrease may be related to nonlinear volume flows observed for short times. This effect is relatively larger at low pressure and it has been related to some mechanical relaxation or strain of the membrane whenever pressure is applied, with a short time scale [24,25]. Accordingly, the effect should be larger for membranes with higher hydraulic permeability, which are in agreement with the results found as shown later.

As it was stated previously, the contribution of the concentration polarization can be eliminated by determining the streaming potential as an intercept at time zero, $\Delta \psi_{0}$, from the plot of the electrical potential difference $\Delta \psi$ vs. $t^{1 / 2}$. Fittings to straight lines to determine the streaming potential $\left(\Delta \psi_{0}\right)$ are also shown in Fig. 1a-e. In each case, the time interval to perform the linear regressions was chosen testing different points as initial time and choosing those which gave a correlation coefficient closer to unity. The fitting errors for $\Delta \psi_{0}$ were lower than to $1 \mu \mathrm{V}$. The estimated values of $\Delta \psi_{0}$ are shown in Fig. 2 as a function of the pressure difference across the membrane. As can be observed, heterogeneous membranes (Fig. 2a) present lower values of the streaming potential than homogeneous membranes (Fig. 2b). Moreover, it is observed that there is a linear dependence between the streaming potential and the applied pressure difference. It then follows that the pressure coefficient $\beta_{0}$, which is defined as follows:

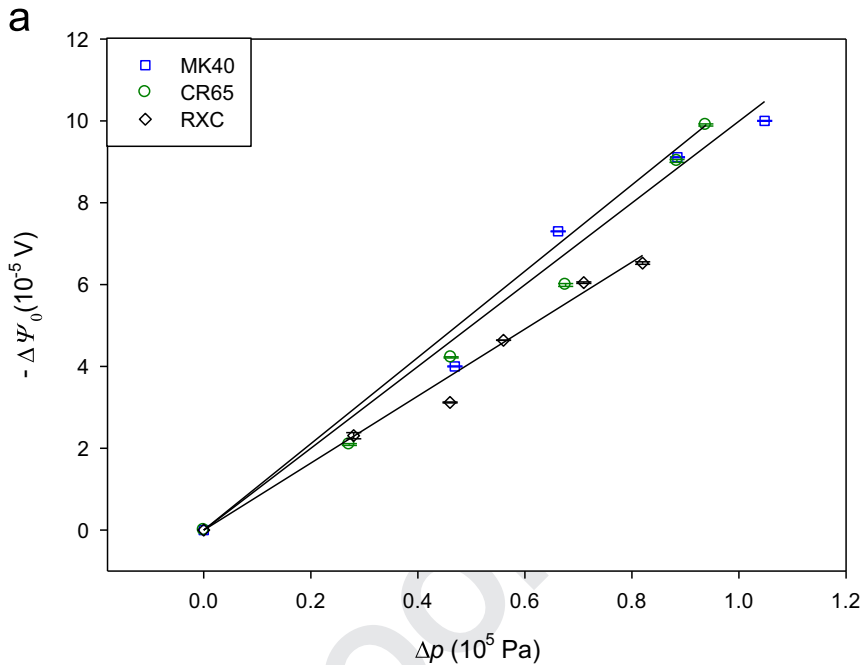

b

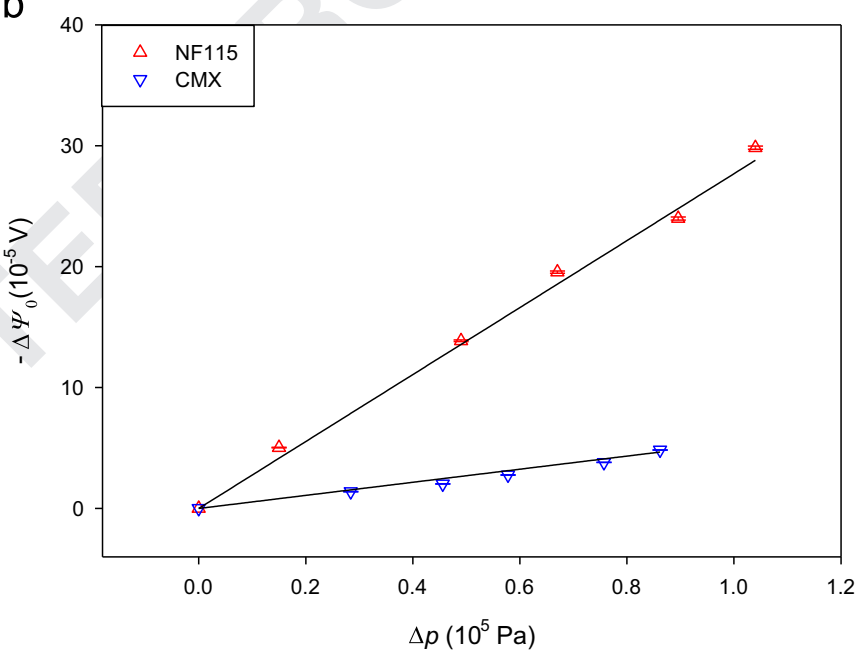

Fig. 2. Time variation of the streaming potential at zero time, $\Delta \psi_{0}$ : (a) heterogeneous membranes and (b) homogeneous membranes.

$\beta_{0} \equiv\left(\frac{\Delta \psi_{0}}{\Delta P}\right)_{I=0}$

is independent of the pressure difference. This fact allows the estimation of this coefficient from the slopes of the straight lines that represent $\Delta \psi_{0}$ vs. $\Delta P$. The obtained values for the adjusted parameter are reported in Table 3. The true value of the pressure coefficient, $\beta_{r}$, can be estimated taking into account the correction given in Eq. (7) as follows:

$\beta_{r}=\beta_{0}-\frac{1}{F}\left(v_{\mathrm{AgCl}}-v_{\mathrm{Ag}}\right)$

\section{Table 3}

Pressure coefficients, $\beta_{0}$ and $\beta_{r}$, electro-osmotic permeability, $W$, and pressurenormalized parameter $A^{*}\left(A^{*} \Xi A / \Delta p\right)$ of cation-exchange membranes used in this study.

\begin{tabular}{llll}
\hline Membrane & $-\beta_{0}\left(10^{-9} \mathrm{~V} / \mathrm{Pa}\right)$ & $\begin{array}{l}-\beta_{r}\left(10^{-9} \mathrm{~V} / \mathrm{Pa}\right) W \\
\left(10^{-9} \mathrm{~m}^{3} / \mathrm{C}\right)\end{array}$ & $\begin{array}{l}A^{*}\left(10^{-10} \mathrm{~V} / \mathrm{s}^{1 /}\right. \\
2 \mathrm{~Pa})\end{array}$ \\
\hline MK40 & $1.00 \pm 0.04$ & $1.15 \pm 0.04$ & $2.11 \pm 0.09$ \\
CR65 & $1.05 \pm 0.05$ & $1.20 \pm 0.05$ & $2.09 \pm 0.06$ \\
RXC & $0.78 \pm 0.03$ & $0.93 \pm 0.03$ & $0.65 \pm 0.16$ \\
CMX & $0.54 \pm 0.03$ & $0.70 \pm 0.03$ & $0.25 \pm 0.06$ \\
NF115 & $2.77 \pm 0.06$ & $2.92 \pm 0.06$ & $1.73 \pm 0.08$ \\
\hline
\end{tabular}

86 
In agreement with Eq. (5), the values of $\beta_{r}$ are equal to the electro-osmotic permeability $W$. The values of $W$ are also given in Table 3. The values of the pressure coefficient are negative because the electric potential difference has an opposite sign than the pressure difference. The estimated electro-osmotic permeabilities using Saxén's law for MK40, CR65 and NF115 are in good agreement with data published elsewhere [26]. The pressure coefficient, and thus, the electro-osmotic permeability, follows the sequence: NF115 $>$ CR65 $\geq$ MK40 $>$ RXC $>$ CMX. It is noted the remarkable high electro-osmotic permeability of the NF115 membrane. As stated above, a factor that influences the electrical properties of the membranes is the liquid content of the membranes. Results on the pressure coefficient in aqueous solutions [27] showed that the magnitude of the pressure coefficient is related to the degree of swelling of the membranes. Membranes with a higher degree of swelling give higher streaming potential values. The results in Tables 2 and 3 confirm the relation between the electro-osmotic permeability of the membranes and their degree of swelling. However, NF115 membrane does not follow this trend because it exhibits the lowest solution uptake and the highest pressure coefficient. The behaviour exhibited by the NF115 membrane may be due to the influence of the solution on the electrical properties of the membrane, because the ion mobility in the membrane depends on the solvent and, so, affects the electrical properties of the membrane. Due to its lower liquid content, this membrane has the highest fixed charge concentration. Thus, it is expected that NF115 has the highest electrical conductivity. If the fixed charge effect and the conductivity effects are not balanced, the streaming potential of the NF115 may be larger than expected. Instead, it may be speculated that the high electro-osmotic permeability of NF115 can be attributed to a structural effect. This membrane is a noncrosslinked membrane, whereas the other membranes are either cross-linked or reinforced. Previous studies reported the physical deformation of some polymeric membranes caused by the application of a hydrostatic pressure difference across them [25]. Results show differences depending on the membrane structure, thus, cross-linked membranes are less susceptible to be deformed due to their rigid structures, in contrast, non-crosslinked membranes, such as NF115, showed significant plastics deformations. In the present study, when the streaming potential measurements are finished, a visual inspection of the membranes revealed that while the NF115 membrane undergoes a significant deformation caused by the application of the pressure difference, the rest of the membranes maintain their shapes. As the influence of the solution on the degree of swelling has less influence for highly cross-linked membranes, it is thus expected that the influence would be greater for the non-crosslinked NF115 membrane. Finally, when membranes of equal character (homogeneous/heterogeneous) are compared, it seems to be a relation between the pressure coefficient and the effective concentration of fixed charged, because higher concentrations lead to higher electro-kinetic coefficients.

Electro-osmosis in the membranes can be also analysed in terms of the so-called electro-osmotic drag coefficient, $k_{w}$. This coefficient describes how many water molecules are carried along with each counter-ion at the vectored counter-ion transport path through the membrane, assuming the gradient of the water concentration is negligible small through the whole membrane. The relation between $W$ and $k_{w}$ is given by the following expression $[5,16,28]$ :

$k_{w}=\frac{F W \rho_{w}}{M_{w}}$

where $\rho_{w}$ and $M_{w}$ are the density and the molecular mass of the transported flow, respectively. The estimated values of $k_{w}$ are reported in Table 4. Previous studies noted that the higher the
Table 4

Water permeability $\left(L_{p}\right)$ and electro-osmotic drag coefficient, $\left(k_{w}\right)$ of cation-exchange membranes used in this study.

\begin{tabular}{llll}
\hline Membrane & $f L_{P}\left(10^{-5} \mathrm{~m}^{3} / \mathrm{s}^{1 / 2} \mathrm{~mol}\right)$ & $L_{P}\left(10^{-13} \mathrm{~m} / \mathrm{s} \mathrm{Pa}\right)$ & $k_{w}$ \\
\hline MK40 & 2.03 & 1.18 & 6 \\
CR65 & 2.01 & 1.16 & 6 \\
RXC & 0.63 & 0.37 & 5 \\
CMX & 0.24 & 0.14 & 4 \\
NF115 & 1.67 & 0.97 & 16 \\
\hline
\end{tabular}

degree of crosslinking of the membrane, the lower is the value for the electro-osmotic drag [28]. Our results support this idea. As it can be observed, the electro-osmotic drag coefficient was higher for non-crosslinked NF115, whereas CMX with high crosslinking degree shows a lower value. As the physical meaning of the water drag coefficient $k_{w}$ is rather close to that of the water transference number $t_{w}$, the comparison of both transport numbers can be a good test to check the reliability of the indirect method presented in this present paper for water transport number evaluation. The water transport number reported for $\mathrm{MK} 40$ in $\mathrm{NaCl}$ solutions ranged from 10 to 5 depending on the salt concentration [7,29], which is very close to the value obtained in the present study. The water transport number for a CMX membrane equilibrated with a $\mathrm{NaCl}$ solution obtained in the present study is the same value as reported by Larchet et al. [7]

Fig. 3a and b shows, respectively, the values of parameter $A$ for heterogeneous and homogeneous membranes which were obtained from the fittings of $\Delta \psi$ vs. $t^{1 / 2}$, as a function the pressure difference across the membranes under different experimental conditions. The fitting errors for $A$ were lower than $1 \mu \mathrm{V} / \mathrm{s}^{1 / 2}$. It is found a linear dependence between the parameter $A$ and the pressure difference, and the values of the corresponding slopes are given in Table 3.

Following Okada et al. [3] the parameter $A$ can be used to estimate the water permeability $L_{p}$ in a given membrane as follows:

$L_{P}=\frac{A}{f \Delta P}$

where $f$ is a factor, which is defined as

$f=8 R T t_{\mathrm{Cl}^{-}} \sqrt{\frac{1}{\pi D_{\mathrm{NaCl}}}}$

where $t_{\mathrm{Cl}}$ is the transference number of $\mathrm{Cl}^{-}$, and is the diffusion coefficient of $\mathrm{NaCl}$. The diffusion coefficient for $\mathrm{NaCl}$ is $1.547 \times 10^{-9} \mathrm{~m}^{2} / \mathrm{s}[30,31]$. Transference number of $\mathrm{Cl}^{-}$in solution, which was estimated from ionic mobility at infinite dilution, is 0.6073 [30,31]. Results for $L_{P} f$ and $L_{P}$ are given in Table 4 . The liquid permeability in the membranes follows the sequence: MK40 $\geq$ CR65 $>$ NF115 $>$ RXC $>$ CMX. It is accepted that the membrane permeability depends on the liquid uptake by the membrane, the interaction between the transported water and ions with the polymer matrix, and the diffusional pathways of the transported species through the membrane, among other factors. The influence of these variables in the permeability can lead to its rise or decrease depending on the relative magnitude of each one. In MK40, CR65 and CMX, the permeability seems to be proportional to the liquid content of the membranes. Thus, the higher membrane liquid uptake, the higher the membrane permeability is. In contrast, in NF115 and RXC the degree to which the liquid swells the membrane is not the most influential factor in the permeability of these two membranes. NF115 exhibits a high membrane permeability considering its low liquid content, and RXC has lower membrane permeability than that expected according to its high liquid content. This behaviour suggests that 


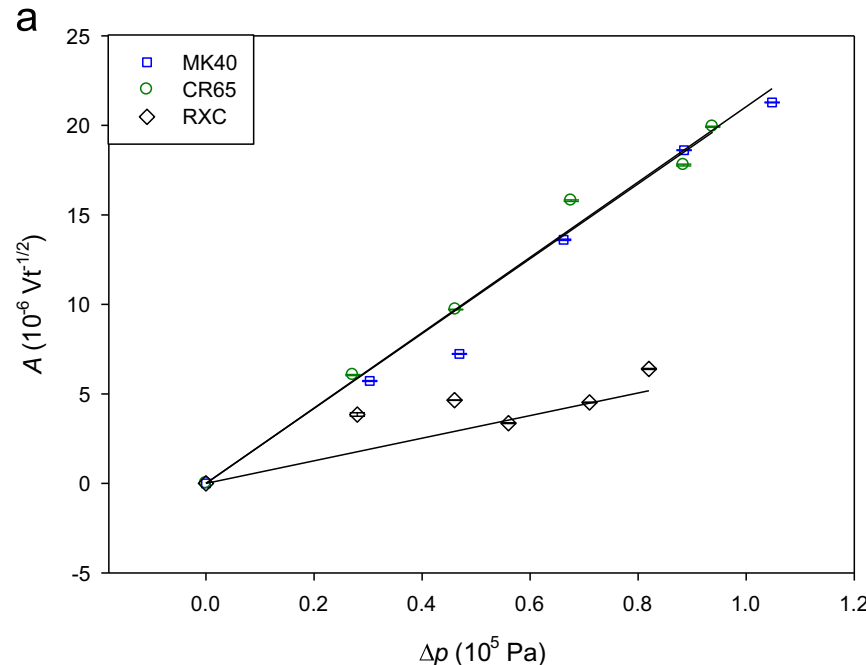

b

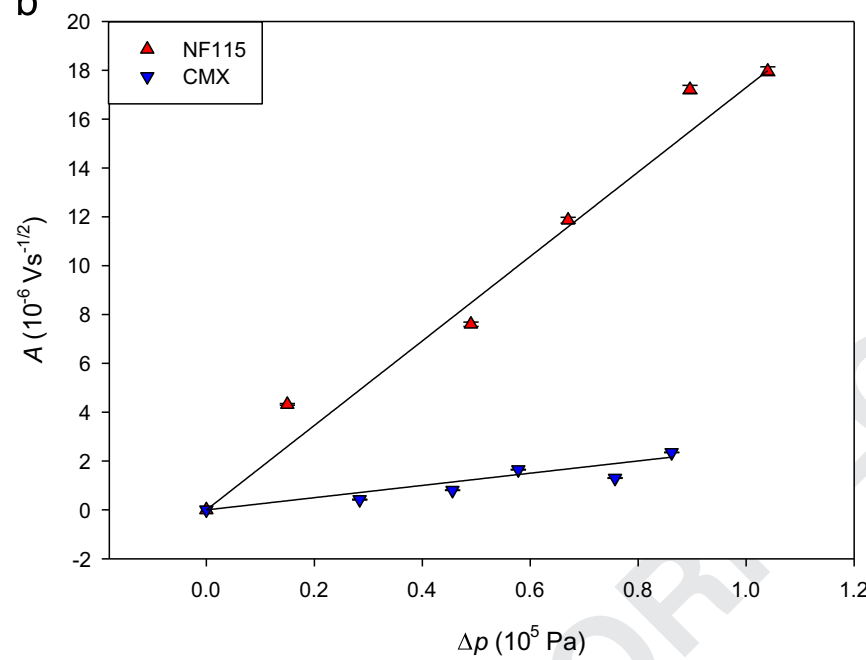

Fig. 3. Parameter $A$ as a function of the pressure difference across the membrane: (a) heterogeneous membranes and (b) homogeneous membranes.

membrane microstructure and the interaction between the transported species and the polymer matrix plays a role in the permeation process across these two membranes. On the other hand, the estimated liquid permeability values $L_{P}$ can be compared to data reported in the literature in order to check the reliability of the method. As an example, we obtained a value of 1.16, in units of $10^{-13} \mathrm{~m} / \mathrm{s}$ Pa, for CR65, whereas Okada et al. [3] reported a value of 2.5. We obtained a value of 0.97 for NF115 and the reported value is 1.1 [32-37].

\section{Conclusions}

Heterogeneous membranes exhibit higher liquid content and wet porosity than homogeneous membranes, indicating that heterogeneous membranes are more hydrophilic. Accordingly, the FH interaction parameters are lower for heterogeneous membranes than that of homogeneous membranes.

In general, the differences between the liquid uptakes of the membranes equilibrated in water and in aqueous $\mathrm{NaCl}$ solutions are not significant. The liquid uptake by the non-crosslinked NF115 membrane equilibrated in the aqueous $0.01 \mathrm{M} \mathrm{NaCl}$ solution decreases with respect to the membrane equilibrated in water. In contrast, the liquid uptake properties of MK40, CR65, RXC and
CMX membranes equilibrated in the aqueous $0.01 \mathrm{M} \mathrm{NaCl}$ solution either remains unchanged or increases a little, compared to the membranes equilibrated with pure water. These facts suggest that not only the osmotic pressure and the ionic forces are relevant in determining the liquid uptake properties of the membranes, but also the structure of the membrane matrix affects their sorption properties; the reinforcement of the membranes discourages their sorption ability.

The higher the liquid content of the membranes, the lower the concentration of fixed charges in the membranes. Then, homogeneous membranes have higher concentration of fixed charges than heterogeneous membranes. In addition, the effective charge concentration in the membranes follows the same sequence as the fixed charge concentration, with the exception of MK40, whose effective charge concentration is significantly larger. This can be related to the fact that this membrane combines a high degree of swelling with a high porosity, which leads to a little ion-pairing effect.

The streaming potential across the membranes increases linearly with the established pressure difference, and it is larger in heterogeneous membranes than in homogeneous ones. Moreover, it is observed that, with the exception of NF115, the higher the membrane liquid content, the higher the streaming potential. The behaviour exhibited by the NF115 membrane may be due to either the influence of the solution on the electrical properties of the membrane, or a structural effect.

In MK40, CR65 and CMX the liquid content of the membrane seems to be the most important factor in the liquid permeability of the membranes. Thus, the higher membrane liquid uptake, the higher the membrane permeability. In NF115 and RXC other factors, such as the diffusional pathways in the membrane or the interactions between the transported components and the polymer membrane, may play an important role in the overall transport process.

\section{Acknowledgements}

The author would like to thank the anonymous reviewers for their valuable comments and suggestions to improve the quality of the paper. Financial support of this work by Banco de Santander and Universidad Complutense de Madrid within the framework of Project PR6/13-18853 is gratefully acknowledged.

\section{References}

[1] R.P. Rastogi, R.C. Srivastava, S.N. Singh, Nonequilibrium thermodynamics of electrokinetic phenomena, Chem. Rev. 93 (1993) 1945-1990.

[2] V.M. Barragán, C. Ruiz-Bauzá, J.L. Imaña, Streaming potential across cationexchange membranes in methanol-water electrolyte solutions, J. Colloid Interface Sci. 294 (2006) 473-481.

[3] T. Okada, S. Kjelstrup Ratkje, H. Hanche-Olsen, Water transport in cation exchange membranes, J. Membr. Sci. 66 (1992) 179-192.

[4] K.S. Spiegler, On the measurement of streaming potential with silver-silver chloride electrodes, Desalination 15 (1974) 135-140.

[5] Y.S. Li, T.S. Zhao, W.W. Yang, Measurements of water uptake and transport properties in anion-exchange membranes, Int. J. Hydrogen Energy 35 (2010) 5656-5665.

[6] V.M. Barragán, C. Ruiz-Bauzá, J.P.G. Villaluenga, B. Seoane, On the methanolwater electroosmotic transport in a Nafion membrane, J. Membr. Sci. 236 (2004) 109-120.

[7] C. Larchet, B. Auclair, V. Nikonenko, Approximate evaluation of water transport number in ion-exchange membranes, Electrochim. Acta 49 (2004) 1711-1717.

[8] J.P.G. Villaluenga, V.M. Barragán, M.A. Izquierdo-Gil, M.P. Godino, B. Seoane, C. Ruiz-Bauzá, Comparative study of liquid uptake and permeation characteristics of sulfonated cation-exchange membranes in water and methanol, J. Membr. Sci. 323 (2008) 421-427.

[9] D. Nandan, H. Mohan, R.M. Iyer, Methanol and water uptake, densities, equivalental volumes and thicknesses of several uni- and divalent ionic 
perfluorosulphonate exchange membranes (Nafion 117) and their methanolwater fractionation behavior at $298 \mathrm{~K}$, J. Membr. Sci. 71 (1992) 69-80.

[10] K.A. Mauritz, R.B. Moore, State of understanding of Nafion, Chem. Rev. 104 (2004) 4535-4586.

[11] E. Güler, R. Elizen, D.A. Vermaas, M. Saakes, K. Nijmeijer, Performance-determining membrane properties in reverse electrodialysis, J. Membr. Sci. 446 (2013) $266-276$

[12] J.-H. Choi, S.-H. Moon, Pore size characterization of cation-exchange membranes by chronopotentiometry using homologous amine ions, J. Membr. Sci. 191 (2001) 225-236.

[13] P. Długołecki, B. Anet, S.J. Metz, K. Nijmeijer, M. Wessling, Transport limitations in ion exchange membranes at low salt concentrations, J. Membr. Sci. 346 (2010) 163-171.

[14] V.M. Barragán, M.J. Pérez-Haro, Correlations between water uptake and effective fixed charge concentration at high univalent electrolyte concentrations in sulfonated polymer cation-exchange membranes with different morphologies, Electrochim. Acta 56 (2011) 8630-8637.

[15] D. García-Nieto, V.M. Barragán, A comparative study of the electro-osmotic behavior of cation and anion exchange membranes in alcohol-water media, Electrochim. Acta 154 (2015) 166-176.

[16] M.H.V. Mulder, Thermodynamic principles of pervaporation, in: R.Y.M. Huang (Ed.), Pervaporation Membrane Separation Processes, Elsevier, Amsterdam, The Netherlands, 1991.

[17] V.M.M. Lobo, Electrolyte Solutions: Literature Data on Thermodynamics and Transport Properties, Coimbra (Ed.), Coimbra, 1975.

[18] F.G. Helfferich, Ion Exchange, Dover, New York, 1995.

[19] A. Lehmani, P. Turq, M. Périé, J. Périé, J.-P. Simonin, Ion transport in Nafion 117 membrane, J. Electroanal. Chem. 428 (1997) 81-89.

[20] E.D. Belashova, N.A. Melnik, N.D. Pismenskaya, K.A. Shevtsova, A.V. Nebvausky, K.A. Ledeveu, V.V. Nikonenko, Electrochim. Acta 59 (2012) 412-423.

[21] B. Piluharto, V. Suendo, T. Ciptati, C.L. Radiman, Strong correlation between membrane effective fixed charge and proton conductivity in the sulfonated polysulfone cation-exchange membranes, Ionics 17 (2011) 229-238.

[22] M.A. Islam, N.D. Nikolov, J.D. Nikolova, An electrical model for the relaxation of streaming potential across a charged membrane, J. Membr. Sci. 84 (1993) $29-36$.

[23] V.M. Barragán, C. Ruiz-Bauzá, Streaming potential and hydraulic permeation through cation-exchange membranes, J. Non-Equilib. Thermodyn. 22 (1997) 374-385.

[24] B.S. Kilsgaard, S. Haldrup, J. Catalano, A. Bentien, High figure of merit for electrokinetic energy conversion in Nafion, J. Power Sources 247 (2014) 235-242.

[25] V.M. Barragán, E. Pastuschuk, Viscoelastic deformation of sulfonated polymeric cation-exchange membranes exposed to a pressure gradient, Mater. Chem. Phys. 146 (2014) 65-72.

[26] V.M. Barragán, J.P.G. Villaluenga, M.P. Godino, M.A. Izquierdo-Gil, C. RuizBauzá, B. Seoane, Swelling and electro-osmotic properties of catión-exchange membranes with different structures in methanol-water media, J. Power Sources 185 (2008) 822-827.

[27] J.M. Reynard, C. Larchet, G. Bulvestre, B. Auclair, Determination of the streaming potential in ion-exchange membranes, J. Membr. Sci. 67 (1992) $57-66$.

[28] T. Yamanaka, T. Takeguchi, H. Takahashi, W. Ueda, Water transport during ion conduction in anion-exchange and cation-exchange membranes, J. Electrochem. Soc. 156 (2009) 13831-13835.

[29] N.P. Berezina, N.A. Kononenko, O.A. Dyomina, N.P. Gnusin, Characterization of ion-exchange membrane materials: properties vs. structure, Adv. Colloid Interface Sci. 139 (2008) 3-28.

[30] B.E. Conway, Electrochemical Data, Greenwood Press, Westport, CT, 1969.

[31] C. Larchet, S. Nouri, B. Auclair, L. Dammak, V. Nikonenko, Application of chronopotentiometry to determine the thickness of diffusion layer adjacent to an ion-exchange membrane under natural convection, Adv. Colloid Interface Sci. 139 (2008) 45-61.

[32] V.M. Barragán, J.P.G. Villaluenga, M.P. Godino, M.A. Izquierdo-Gil, C. RuizBauzá, B. Seoane, Experimental estimation of equilibrium and transport properties of sulfonated cation-exchange membranes with different morphologies, J. Colloid Interface Sci. 333 (2009) 497-507.

[33] J.P.G. Villaluenga, B. Seoane, V.M. Barragán, C. Ruiz-Bauzá, Permeation of electrolyte water-methanol solutions through a Nafion membrane, J. Colloid Interface Sci. 268 (2003) 476-481.

[34] J.P.G. Villaluenga, V.M. Barragán, B. Seoane, C. Ruiz-Bauzá, Sorption and permeation of solutions of chloride salts, water and methanol in a Nafion membrane, Electrochim. Acta 51 (2006) 6297-6303.

[35] S. Koter, The equivalent pore radius of charged membranes from electroosmotic flow, J. Membr. Sci. 166 (2000) 127-135.

[36] S. Koter, W. Kujawski, I. Koter, Importance of the cross-effects in the transport through ion-exchange membranes, J. Membr. Sci. 297 (2007) 226-235.

[37] P.W. Majsztrik, M.B. Satterfield, A.B. Bocarsly, J.B. Benzinger, Water sorption, desorption and transport in Nafion membranes, J. Membr. Sci. 301 (2007) 93-106. 